Mathematical Research Letters 10, 459-467 (2003)

\title{
COMPLEX HOMOGENEOUS SPACES OF PSEUDO-RESTRICTED GROUPS
}

\author{
DANiel Beltiţă
}

\begin{abstract}
One constructs invariant Kähler structures on homogeneous spaces of certain classes of infinite-dimensional Lie groups. The main new classes of examples which fall under these general constructions are homogeneous spaces of certain Banach-Lie groups described by means of admissible pairs of ideals of compact operators on Hilbert spaces.
\end{abstract}

\section{Introduction}

The celebrated Borel-Weil Theorem has been recently extended to infinite-dimensional Lie groups modeled on real $L^{*}$-algebras (see [Ne00] and also [Ne01]). We recall (cf. [Ba72]) that the latter object is a real involutive Banach-Lie algebra $\mathfrak{X}$ whose underlying Banach space is in fact a Hilbert space with the scalar product $(\cdot \mid \cdot)$ satisfying

$$
(\forall x, y, z \in \mathfrak{X}) \quad([x, y] \mid z)=\left(y \mid\left[x^{*}, z\right]\right) .
$$

A key step in the aforementioned extension is the construction of invariant Kähler structures on coadjoint orbits of the infinite-dimensional Lie groups under consideration.

It is the aim of the present note to construct Kähler homogeneous spaces of certain Banach-Lie groups which are more general than the $L^{*}$-groups of [Ne00], thus suggesting that there might exist an interesting representation theory for some new classes of infinite-dimensional Lie groups, which we call pseudorestricted groups (see Definitions 2.1 and 2.4 below). These include in particular groups constructed from ideals of compact operators which were less employed in Lie theory until now, more specifically Lorentz ideals.

In the present research, we keep close to the traditional method to study homogeneous spaces by working mainly on Lie algebra level (cf. e.g., [DN88]). To pass beyond the realm of $L^{*}$-groups, we have to avoid two difficulties: firstly, the existence of non-enlargible Banach-Lie algebras (see e.g., [EK64]), and secondly,

Received October 1, 2002.

Key words and phrases. Banach-Lie group, complex polarization, Kähler structure, spectral measure, operator ideal.

2000 Mathematics Subject Classification. Primary 58B12; Secondary 22E65, 32M10, 53C55, $47 \mathrm{~L} 20$. 
the fact that a closed subalgebra of a Banach-Lie algebra may have no closed complement (which is a key point in order to endow homogeneous spaces with charts making them into smooth manifolds). It turns out that both these points are most conveniently settled in our approach, which eventually leads to new classes of infinite-dimensional Kähler homogeneous spaces.

The main achievements of this work are:

1. a new enlargibility criterion for Banach-Lie algebras (Theorem 2.3), which actually concerns all central extensions of the algebra under consideration;

2. a method to construct Kähler polarizations of Banach-Lie algebras in certain vector-valued cocycles (Theorem 3.2); in other words, that method produces a complex polarization which works for several given cocycles, which extends the main result of [Be01];

3. a new class of infinite-dimensional Kähler homogeneous spaces, constructed from ideals of compact operators on Hilbert spaces (see Theorem 4.4 and Example 4.3).

We now introduce some notation. For Banach spaces $\mathfrak{X}$ and $\mathfrak{Y}$ over $\mathbb{K} \in\{\mathbb{R}, \mathbb{C}\}$, we denote by $\mathfrak{X}^{\#}$ and $\mathfrak{Y}^{\#}$ their topological duals, respectively, and by $T^{\#}: \mathfrak{Y} \# \rightarrow$ $\mathfrak{X}^{\#}$ the dual of any $T \in \mathcal{B}(\mathfrak{X}, \mathfrak{Y})$ (the bounded linear operators from $\mathfrak{X}$ into $\mathfrak{Y}$ ). If $\mathfrak{g}$ is a real involutive Banach-Lie algebra, we denote by $\mathcal{C}_{0}^{+}(\mathfrak{g})$ the set of all $\iota \in \mathcal{B}\left(\mathfrak{g}^{\#}, \mathfrak{g}\right)$ such that

(a) $\iota^{\#}=\iota$ (where we view $\mathfrak{g} \subseteq\left(\mathfrak{g}^{\#}\right)^{\#}$ as usual) and Ker $\iota=\{0\}$,

(b) for all $f \in \mathfrak{g}^{\#}$ we have $\langle f, \iota(f)\rangle \geq 0$ (where $\langle\cdot, \cdot\rangle: \mathfrak{g}^{\#} \times \mathfrak{g} \rightarrow \mathbb{R}$ is the duality pairing), and

(c) for every $a \in \mathfrak{g}$ we have $\left(\operatorname{ad}_{\mathfrak{g}} a\right) \circ \iota=\iota \circ\left(\operatorname{ad}_{\mathfrak{g}} a^{*}\right)^{\#}$.

The elements of $\mathcal{C}_{0}^{+}(\mathfrak{g})$ are called (injective) equivariant monotone operators.

If $\mathfrak{A}$ is a complex Banach space and $\mathfrak{z}_{0}$ is a real Banach space, we say that $\Psi \in \mathcal{B}\left(\mathfrak{z}_{0}, \mathcal{B}(\mathfrak{A})\right)$ is a hermitian map if $\|\exp (i \Psi(\gamma))\| \leq 1$ for all $\gamma \in \mathfrak{z}_{0}$.

For every real or complex Banach-Lie algebra $\mathfrak{Y}$ we denote by $\operatorname{Der}(\mathfrak{Y})$ the Banach-Lie algebra of its bounded derivations. For a real Banach-Lie algebra $\mathfrak{g}$, the canonical involution is defined by $a \mapsto-a$. A compact $L^{*}$-algebra is a real $L^{*}$-algebra whose involution is just the canonical one. For every real involutive Banach-Lie algebra $\mathfrak{g}$ we denote by $\mathfrak{g}_{\mathbb{C}}$ its complexification and, if it

is not otherwise stated, we denote by $a \mapsto \bar{a}$ the conjugation of $\mathfrak{g}_{\mathbb{C}}$ whose set of fixed points is just $\mathfrak{g}$.

We conclude this Introduction by mentioning that proofs for all of the theorems in the present note can be found in [Be02a].

\section{Pseudo-restricted groups}

The terminology of the following definition claims its origin from the restricted groups occurring in the theory of loop groups (see [PS90]). 
Definition 2.1. Let $\mathbb{K} \in\{\mathbb{R}, \mathbb{C}\}$ and $\mathfrak{Y}$ a Banach-Lie algebra over $\mathbb{K}$. Also let $\mathfrak{z}_{0}$ be a real Banach space. Then to every $\Psi \in \mathcal{B}\left(\mathfrak{z}_{0}, \operatorname{Der}(\mathfrak{Y})\right)$ and $\iota \in \mathcal{B}\left(\mathfrak{Y}^{\#}, \mathfrak{Y}\right)$ with $\operatorname{Ker} \iota=\{0\}$ and $[\operatorname{Ran} \iota, \mathfrak{Y}] \subseteq \operatorname{Ran} \iota$ we associate the pseudo-restricted algebra

$$
\mathfrak{Y}(\Psi, \iota):=\bigcap_{\gamma \in \mathfrak{z} 0} \Psi(\gamma)^{-1}(\operatorname{Ran} \iota)
$$

endowed with the norm defined by

$$
(\forall y \in \mathfrak{Y}(\Psi, \iota)) \quad\|y\|_{\mathfrak{Y}(\Psi, \iota)}=\|y\|_{\mathfrak{Y}}+\left\|\iota^{-1}(\Psi(\cdot) y)\right\|_{\mathcal{B}(\mathfrak{z} 0, \mathfrak{Y} \#)} .
$$

Then $\mathfrak{Y}(\Psi, \iota)$ is a subalgebra of $\mathfrak{Y}$ which is a Banach-Lie algebra in its own right with respect to the norm $\|\cdot\|_{\mathfrak{Y}(\Psi, \iota)}$.

In the same setting, it makes sense to define

$$
\omega_{\Psi, \iota}: \mathfrak{Y}(\Psi, \iota) \times \mathfrak{Y}(\Psi, \iota) \rightarrow\left(\mathfrak{z}_{0}\right)^{\#}, \quad\left(y_{1}, y_{2}\right) \mapsto\left\langle\iota^{-1}\left(\Psi(\cdot) y_{1}\right), y_{2}\right\rangle .
$$

Theorem 2.2. Let $\mathfrak{g}$ be a real Banach-Lie algebra equipped with its canonical involution, $\mathfrak{z}_{0}$ a real Banach space, and $\iota \in \mathcal{C}_{0}^{+}(\mathfrak{g})$. Assume that $\Psi: \mathfrak{z}_{0} \rightarrow \operatorname{Der}(\mathfrak{g})$ has the property that $i \Psi \in \mathcal{B}\left(\mathfrak{z}_{0}, \mathcal{B}\left(\mathfrak{g}_{\mathbb{C}}\right)\right)$ is a hermitian map,

$$
\left(\forall \gamma \in \mathfrak{z}_{0}\right) \quad \Psi(\gamma) \circ \iota=-\iota \circ \Psi(\gamma)^{\#},
$$

and the Banach space underlying $\mathfrak{g}$ is reflexive. Then $\omega_{\Psi, \iota}$ is a $\left(\mathfrak{z}_{0}\right)^{\#}$-valued continuous 2-cocycle of the real Banach-Lie algebra $\mathfrak{g}(\Psi, \iota)$, and

$$
\mathfrak{h}_{\Psi, \iota}:=\bigcap_{\gamma \in \mathfrak{z}_{0}} \operatorname{Ker} \Psi(\gamma)=\left\{a \in \mathfrak{g}(\Psi, \iota) \mid(\forall b \in \mathfrak{g}(\Psi, \iota)) \quad \omega_{\Psi, \iota}(a, b)=0\right\} .
$$

If moreover $\operatorname{dim} \mathfrak{z}_{0}<\infty$, then $\mathfrak{h}_{\Psi, \iota}$ has a closed complement both in $\mathfrak{g}$ and in $\mathfrak{g}(\Psi, \iota)$.

The proof of the next result makes essential use of the enlargibility criterion of [Pe88] and [Pe92]. (See [Be02b] for a "standard" proof of that criterion.)

Theorem 2.3. If $\mathfrak{g}$ is a real involutive Banach-Lie algebra with $\mathcal{C}_{0}^{+}(\mathfrak{g}) \neq \emptyset$, then every Banach-Lie algebra which is a linearly split central extension of either $\mathfrak{g}$ or $\mathfrak{g}_{\mathbb{C}}$ is enlargible.

Now, by Theorem 2.3 and the fact that a Banach-Lie algebra which is continuously embedded into an enlargible Banach-Lie algebra is in turn enlargible (see assertion $(* * *)$ at page 22 in [EK64]), we can make the following definition.

Definition 2.4. In the setting of Theorem 2.2, every Banach-Lie group whose Lie algebra is topologically isomorphic to the pseudo-restricted algebra $\mathfrak{g}(\Psi, \iota)$ is said to be a pseudo-restricted group associated to the triple $(\mathfrak{g}, \iota, \Psi)$. 


\section{Kähler polarizations}

In order to construct complex structures on homogeneous spaces of pseudorestricted groups (see Theorem 4.4), we need Kähler polarizations in the sense of the following definition which extends the usual notion (see e.g., [Ne00]) to vector-valued cocycles.

Definition 3.1. Let $\mathfrak{g}$ be a real Banach-Lie algebra, $\mathfrak{z}$ a real Banach space and $\omega$ a $\mathfrak{z}$-valued continuous 2-cocycle of $\mathfrak{g}$. Denote

$$
\mathfrak{h}:=\{a \in \mathfrak{g} \mid \omega(a, \mathfrak{g})=\{0\}\} .
$$

Then a (weak) Kähler polarization of $\mathfrak{g}$ in $\omega$ is a closed subspace $\mathfrak{p}$ of $\mathfrak{g}_{\mathbb{C}}$ such that $\mathfrak{p}$ has a closed complement in $\mathfrak{g}_{\mathbb{C}}$ and the following conditions are satisfied.

$(\mathrm{C} 1) \mathfrak{p}$ is a subalgebra of $\mathfrak{g}_{\mathbb{C}}$.

(C2) $\mathfrak{p} \cap \overline{\mathfrak{p}}=\mathfrak{h}+i \mathfrak{h}$.

(C3) $\mathfrak{p}+\overline{\mathfrak{p}}=\mathfrak{g}_{\mathbb{C}}$.

(C4) $\omega(\mathfrak{p}, \mathfrak{p})=\{0\}$.

(C5) There exists a closed convex cone $\Gamma$ in $\mathfrak{z}$ such that $\Gamma \cap(-\Gamma)=\{0\}$ and

$$
(\forall a \in \mathfrak{p} \backslash(\mathfrak{h}+i \mathfrak{h})) \quad-i \omega(a, \bar{a}) \in \Gamma \backslash\{0\}
$$

If moreover the injective operator

$$
\mathfrak{g} / \mathfrak{h} \rightarrow \mathcal{B}(\mathfrak{g} / \mathfrak{h}, \mathfrak{z}), \quad a+\mathfrak{h} \mapsto \omega(a, \cdot)
$$

is invertible, then $\mathfrak{p}$ is said to be a strong Kähler polarization of $\mathfrak{g}$ in $\omega$.

We now state our main theorem concerning existence of Kähler polarizations. The idea of joint spectrum (see e.g., [BS01]) is essentially involved here, under the disguise of the support of a (joint) spectral measure.

Theorem 3.2. Let $\mathfrak{g}$ be a real Banach-Lie algebra equipped with its canonical involution, $\iota \in \mathcal{C}_{0}^{+}(\mathfrak{g})$ and $\mathfrak{z}$ a finite-dimensional real vector space. Assume that

$$
\Psi: \mathfrak{z}^{\#} \rightarrow \operatorname{Der}(\mathfrak{g})
$$

has the property that, for some spectral measure $E$ on $\mathfrak{z}$ with values in $\mathcal{B}\left(\mathfrak{g}_{\mathbb{C}}\right)$, we have

$$
\left(\forall \gamma \in \mathfrak{z}^{\#}\right) \quad i \Psi(\gamma)=\int_{\mathfrak{z}} \gamma(z) d E(z) \quad \text { and } \quad \Psi(\gamma) \circ \iota=-\iota \circ \Psi(\gamma)^{\#} .
$$

Moreover, assume that the Banach space underlying $\mathfrak{g}$ is reflexive and there exists a compact convex set $C$ in $\mathfrak{z}$ such that $(-C) \cap C=\emptyset$ and

$$
\operatorname{supp} E \subseteq(-C) \cup\{0\} \cup C .
$$


Then the following assertions hold.

(i) The Banach-Lie algebra $\mathfrak{g}$ can be endowed with an equivalent norm such that the map $i \Psi: \mathfrak{z}^{\#} \rightarrow \mathcal{B}\left(\mathfrak{g}_{\mathbb{C}}\right)$ is hermitian.

(ii) If $\widetilde{\mathfrak{A}}$ denotes the complexification of the pseudo-restricted algebra $\mathfrak{g}(\Psi, \iota)$, then $\widetilde{\mathfrak{A}}$ is invariant to $E(\delta)$ for every Borel subset $\delta$ of $\mathfrak{z}$ and

$$
\widetilde{E}(\cdot)=\left.E(\cdot)\right|_{\mathfrak{\mathfrak { A }}}
$$

is a spectral measure on $\mathfrak{z}$ with values in $\mathcal{B}(\widetilde{\mathfrak{A}})$;

(iii) The subspace $\mathfrak{p}:=\operatorname{Ran} \widetilde{E}((-C) \cup\{0\})$ is a Kähler polarization of $\mathfrak{g}(\Psi, \iota)$ in $\omega_{\Psi, \iota}$.

In the following corollary to Theorem 3.2 , for a compact $L^{*}$-algebra $\mathfrak{g}$ with the scalar product $(\cdot \mid \cdot)$, by the canonical equivariant monotone operator $\iota$ of $\mathfrak{g}$ we mean the invertible operator $\iota: \mathfrak{g}^{\#} \rightarrow \mathfrak{g}$ whose inverse is defined by $\iota^{-1}(a)=(a \mid \cdot)$ for all $a \in \mathfrak{g}$. Note that indeed $\iota \in \mathcal{C}_{0}^{+}(\mathfrak{g})$.

Corollary 3.3. Let $\mathfrak{g}$ be a compact $L^{*}$-algebra $\mathfrak{g}$ with the canonical equivariant monotone operator $\iota$. Assume that $\mathfrak{z}$ is a finite-dimensional real vector space and $\Psi: \mathfrak{z}^{\#} \rightarrow \operatorname{Der}(\mathfrak{g})$ is a linear map whose range is a commuting set of skewsymmetric derivations of $\mathfrak{g}$. Then there exists a unique spectral measure $E$ on $\mathfrak{z}$ with values orthogonal projections on the complex Hilbert space $\mathfrak{g}_{\mathbb{C}}$ such that

$$
\left(\forall \gamma \in \mathfrak{z}^{\#}\right) \quad i \Psi(\gamma)=\int_{\mathfrak{z}} \gamma(z) d E(z) .
$$

Moreover,

$$
\omega_{\Psi}: \mathfrak{z} \times \mathfrak{z} \rightarrow\left(\mathfrak{z}^{\#}\right)^{\#}=\mathfrak{z}, \quad(a, b) \mapsto(a \mid \Psi(\cdot) b),
$$

is a $\mathfrak{z}$-valued continuous 2-cocycle of $\mathfrak{g}$ and, if there exists a compact convex subset $C$ of $\mathfrak{z}$ such that $(-C) \cap C=\emptyset$ and

$$
\operatorname{supp} E \subseteq(-C) \cup\{0\} \cup C,
$$

then the following assertions hold.

(i) The subspace $\mathfrak{p}:=\operatorname{Ran} E((-C) \cup\{0\})$ is a Kähler polarization of $\mathfrak{g}$ in $\omega_{\Psi}$.

(ii) If $\gamma \in \mathfrak{z}^{\#}$ has the property that $\gamma(C) \subseteq(0, \infty)$, then $\mathfrak{p}$ is a strong Kähler polarization of $\mathfrak{g}$ in the $\mathbb{R}$-valued continuous 2 -cocycle $\gamma \circ \omega_{\Psi}$.

\section{Homogeneous spaces and operator ideals}

Throughout the present section, $\mathfrak{H}$ stands for a complex Hilbert space with the scalar product denoted by $(\cdot \mid \cdot)$. In the next definition, we use the notion of Banach-Lie subgroup in the sense of [Up85]. 
Definition 4.1. If $\mathfrak{z}$ is a real Banach space and $U$ is a real Banach-Lie group, then a weakly (respectively, strongly) Kähler homogeneous space of $U$ of type $\mathfrak{z}$ is a triple $(M, \Omega, I)$ satisfying the following conditions.

(a) There exists a Banach-Lie subgroup $H$ of $U$ with $M=U / H$, and $\Omega$ is a closed $\mathfrak{z}$-valued smooth 2 -form on the manifold $M$, such that $\Omega$ is invariant under the natural action of $U$ on $M$ and is non-degenerate in the sense that, for every $m \in M$, the operator

$$
\alpha_{m}: T_{m} M \rightarrow \mathcal{B}\left(T_{m} M, \mathfrak{z}\right), \quad v \mapsto \Omega_{m}(v, \cdot),
$$

is injective (respectively, for every $m \in M$, the operator $\alpha_{m}$ is bijective).

(b) The symbol $I$ stands for a $U$-invariant complex structure on the manifold $M$ such that, for every $m \in M$, we have

$$
\left(\forall v, w \in T_{m} M\right) \quad \Omega_{m}\left(I_{m} v, I_{m} w\right)=\Omega_{m}(v, w),
$$

where $I_{m}: T_{m} M \rightarrow T_{m} M$ is the multiplication-by- $i$ operator.

(c) There exists a closed convex cone $\Gamma$ in $\mathfrak{z}$ such that $\Gamma \cap(-\Gamma)=\{0\}$ and

$$
\left(\forall v \in T_{m} M \backslash\{0\}\right) \quad \Omega_{m}\left(v, I_{m} v\right) \in \Gamma \backslash\{0\}
$$

for all $m \in M$.

The following definition describes a specific setting where we can obtain complex homogeneous spaces by means of Theorem 3.2 (see Theorem 4.4 and Corollary 4.5). We should point out that, in order to make things clear, this definition requires all of the properties needed in Theorem 4.4. Though, some of these properties follow by the others (see e.g., $\S 1$ in Chapter III in [GK69]).

Definition 4.2. An admissible pair of ideals of $\mathcal{B}(\mathfrak{H})$ is a pair $\left(\mathfrak{J}_{0}, \mathfrak{J}_{1}\right)$ of twosided self-adjoint ideals of $\mathcal{B}(\mathfrak{H})$ satisfying the following conditions.

(a) The ideal $\mathfrak{J}_{0}$ is equipped with a norm $\|\cdot\|_{\mathfrak{J}_{0}}$ making it into a reflexive Banach space.

(b) For all $A, B \in \mathcal{B}(\mathfrak{H})$ and $T \in \mathfrak{J}_{0}$ we have

$$
\|T\| \leq\|T\|_{\mathfrak{J}_{0}}=\left\|T^{*}\right\|_{\mathfrak{J}_{0}} \quad \text { and } \quad\|A T B\|_{\mathfrak{J}_{0}} \leq\|A\| \cdot\|T\|_{\mathfrak{J}_{0}} \cdot\|B\| .
$$

For all $x, y \in \mathfrak{H}$ we have $x(\cdot \mid y) \in \mathfrak{J}_{0}$ and $\|x(\cdot \mid y)\|_{\mathfrak{J}_{0}}=\|x\| \cdot\|y\|$.

(c) We have $\mathfrak{J}_{0} \cdot \mathfrak{J}_{1}+\mathfrak{J}_{1} \cdot \mathfrak{J}_{0} \subseteq \mathcal{C}_{1}(\mathfrak{H})$ and $\operatorname{Tr}(T K)=\operatorname{Tr}(K T)$ whenever $T \in \mathfrak{J}_{0}$ and $K \in \mathfrak{J}_{1}$. Moreover, the bilinear functional

$$
\mathfrak{J}_{1} \times \mathfrak{J}_{0} \rightarrow \mathbb{C}, \quad(K, T) \mapsto \operatorname{Tr}(K T),
$$

induces a vector space isomorphism of $\mathfrak{J}_{1}$ onto the topological dual of the Banach space $\left(\mathfrak{J}_{0},\|\cdot\|_{\mathfrak{J}_{0}}\right)$.

(d) We have $\mathfrak{J}_{1} \subseteq \mathfrak{J}_{0}$.

The following example of admissible pair of ideals is based on duality theory for Lorentz ideals (see [GL74] and [Co88]). 
Example 4.3. Recall that, for all $p, q \in(0, \infty)$, the corresponding Lorentz ideal is defined by

$$
\mathcal{C}_{q, p}=\left\{T \in \mathcal{C}_{\infty}(\mathfrak{H}) \mid\|T\|_{q, p}:=\left(\sum_{n=1}^{\infty} \frac{1}{n^{1-\frac{p}{q}}} s_{n}(T)^{p}\right)^{1 / p}<\infty\right\}
$$

where $\mathcal{C}_{\infty}(\mathfrak{H})$ is the set of all compact operators on $\mathfrak{H}$ and, for $T \in \mathcal{C}_{\infty}(\mathfrak{H})$, $\left\{s_{n}(T)\right\}_{n \geq 1}$ stands for the sequence of singular values of $T$. (In particular, if $p=q$, then $\mathcal{C}_{q, p}$ coincides with the Schatten-von Neumann class $\mathcal{C}_{p}$.) Then $\left(\mathcal{C}_{q_{0}, p_{0}}, \mathcal{C}_{q_{1}, p_{1}}\right)$ is an admissible pair of ideals of $\mathcal{B}(\mathfrak{H})$, and one can take $\|\cdot\|_{q_{0}, p_{0}}$ as the needed norm on $\mathcal{C}_{q_{0}, p_{0}}$, provided $2 \leq p_{0} \leq q_{0}<\infty$ and $\frac{1}{p_{1}}+\frac{1}{p_{0}}=$ $\frac{1}{q_{1}}+\frac{1}{q_{0}}=1$.

In the next statement, for a two-sided self-adjoint ideal $\mathfrak{J}$ of $\mathcal{B}(\mathfrak{H})$, we denote $\mathfrak{u}_{\mathfrak{J}}=\left\{T \in \mathfrak{J} \mid T^{*}=-T\right\}$ and $\mathrm{U}_{\mathfrak{J}}=\mathrm{U}(\mathfrak{H}) \cap\left(\mathrm{id}_{\mathfrak{H}}+\mathfrak{J}\right)$, where $\mathrm{U}(\mathfrak{H})$ is the group of unitary operators on $\mathfrak{H}$. We note that, when $\mathfrak{J}$ is equipped with a complete norm which is stronger than the operator norm, the set $U_{\mathfrak{J}}$ has a natural structure of real Banach-Lie group with the Lie algebra $\mathfrak{u}_{\mathfrak{J}}$, such that the inclusion map $\mathrm{U}_{\mathfrak{J}} \hookrightarrow \mathrm{U}(\mathfrak{H})$ is a homomorphism of real Banach-Lie groups. We also recall the notation $\mathcal{S}^{\prime}$ for the commutant of some $\mathcal{S} \subseteq \mathcal{B}(\mathfrak{H})$ (i.e., $\mathcal{S}^{\prime}$ is the set of all bounded linear operators on $\mathfrak{H}$ which commute with each element of $\mathcal{S}$ ).

Theorem 4.4. Let $\left(\mathfrak{J}_{0}, \mathfrak{J}_{1}\right)$ be an admissible pair of ideals of $\mathcal{B}(\mathfrak{H}), \iota: \mathfrak{u}_{\mathfrak{J}_{1}} \rightarrow \mathfrak{u}_{\mathfrak{J}_{0}}$ the inclusion map, $n \geq 1$, and $A=\left(A_{1}, \ldots, A_{n}\right)$ a commuting $n$-tuple of bounded self-adjoint operators on $\mathfrak{H}$. Define

$$
\Psi: \mathbb{R}^{n} \rightarrow \operatorname{Der}\left(\mathfrak{u}_{\mathfrak{J}_{0}}\right), \quad\left(t_{1}, \ldots, t_{n}\right) \mapsto \operatorname{ad}_{\mathfrak{u}_{\mathfrak{J}_{0}}}\left(i\left(t_{1} A_{1}+\cdots+t_{n} A_{n}\right)\right),
$$

and then

$$
\mathrm{U}_{\mathfrak{J}_{0}, \mathfrak{J}_{1}}(A):=\left\{T \in \mathrm{U}_{\mathfrak{J}_{0}} \mid\left[A_{j}, T\right] \in \mathfrak{J}_{1} \text { for } j=1, \ldots, n\right\}
$$

and

$$
H:=\mathrm{U}_{\mathfrak{J}_{0}, \mathfrak{J}_{1}}(A) \cap\left\{A_{1}, \ldots, A_{n}\right\}^{\prime} .
$$

Then the following assertions hold.

(i) The set $\mathrm{U}_{\mathfrak{J}_{0}, \mathfrak{J}_{1}}(A)$ has a structure of real Banach-Lie group which is a pseudo-restricted group associated to the triple $\left(\mathfrak{u}_{\mathfrak{J}_{0}}, \iota, \Psi\right)$, and the inclusion map

$$
\mathrm{U}_{\mathfrak{J}_{0}, \mathfrak{J}_{1}}(A) \hookrightarrow \mathrm{U}_{\mathfrak{J}_{0}}
$$

is a homomorphism of Banach-Lie groups.

(ii) The set $H$ is a Banach-Lie subgroup of $\mathrm{U}_{\mathfrak{J}_{0}, \mathfrak{J}_{1}}(A)$ with the Lie algebra

$$
\mathfrak{h}:=\mathfrak{u}_{\mathfrak{J}_{0}} \cap\left\{A_{1}, \ldots, A_{n}\right\}^{\prime}
$$


(iii) The $\mathbb{R}^{n}$-valued 2-cocycle $\omega_{\Psi, \iota}$ of the pseudo-restricted algebra $\mathfrak{u}_{\mathfrak{J}_{0}}(\Psi, \iota)$ induces a closed $\mathbb{R}^{n}$-valued smooth 2 -form $\Omega$ on the manifold $\mathrm{U}_{\mathfrak{J}_{0}, \mathfrak{J}_{1}}(A) / H$, which is $\mathrm{U}_{\mathfrak{J}_{0}, \mathfrak{J}_{1}}(A)$-invariant and non-degenerate.

(iv) If the spectra of all of the operators $A_{1}, \ldots, A_{n}$ are finite, then there exists a complex structure $I$ on the manifold $\mathrm{U}_{\mathfrak{J}_{0}, \mathfrak{J}_{1}}(A) / H$ such that the triple

$$
\left(\mathrm{U}_{\mathfrak{J}_{0}, \mathfrak{J}_{1}}(A) / H, \Omega, I\right)
$$

is a weakly Kähler homogeneous space of $\mathrm{U}_{\mathfrak{J}_{0}, \mathfrak{J}_{1}}(A)$ of type $\mathbb{R}^{n}$.

The following corollary is obtained by means of Corollary 3.3, and extends Theorem VII.6 in [Ne00].

Corollary 4.5. In the setting of Theorem 4.4 (iv), if $\mathfrak{J}_{0}=\mathfrak{J}_{1}=\mathcal{C}_{2}$ (the HilbertSchmidt class) and $\gamma \in\left(\mathbb{R}^{n}\right)^{\#}$ such that

$$
\operatorname{Ker} \gamma \cap\left\{\left(t_{1}-s_{1}, \ldots, t_{n}-s_{n}\right) \mid t_{j}, s_{j} \in \sigma\left(A_{j}\right) \text { for } j=1, \ldots, n\right\}=\{0\} \text {, }
$$

then the triple $\left(\mathrm{U}_{\mathcal{C}_{2}} / H, \gamma \circ \Omega, I\right)$ is a strongly Kähler homogeneous space of $\mathrm{U}_{\mathcal{C}_{2}}$ of type $\mathbb{R}$.

\section{Acknowledgment}

We thank the referee for a most careful reading of the manuscript and for a number of useful remarks.

\section{References}

[Ba72] V. K. Balachandran, Real L*-algebras, Indian J. Pure Appl. Math. 3 (1972), 12241246.

[Be01] D. Beltiţă, On Banach-Lie algebras, spectral decompositions and complex polarizations, preprint, 2001.

[Be02a] _ Equivariant monotone operators and infinite-dimensional complex homogeneous spaces, preprint, 2002.

[Be02b] _ Asymptotic products and enlargibility of Banach-Lie algebras, to appear in J. Lie Theory.

[BS01] D. Beltiţă, M. Şabac, Lie algebras of bounded operators, Operator Theory: Advances and Applications, 120. Birkhäuser Verlag, Basel, 2001.

[Co88] F. Cobos, Duality and Lorentz-Marcinkiewicz operator spaces, Math. Scand. 63 (1988), 261-267.

[DN88] J. Dorfmeister, K. Nakajima, The fundamental conjecture for homogeneous Kähler manifolds, Acta Math. 161 (1988), 23-70.

[EK64] W. T. van Est, Th. J. Korthagen, Non-enlargible Lie algebras, Nederl. Akad. Wetensch. Proc. Ser. A 67=Indag. Math. 26 (1964), 15-31.

[GL74] Ch. Gapaillard, P. T. Lai, Remarques sur les propriétés de dualité et d'interpolation des idéaux de R. Schatten, Studia Math. 49 (1973/74), 129-138.

[GK69] I. C. Gohberg, M. G. Krĕn, Introduction to the theory of linear nonselfadjoint operators, Translated from the Russian by A. Feinstein. Translations of Mathematical Monographs, Vol. 18. American Mathematical Society, Providence, RI, 1969. 
[Ne00] K.-H. Neeb, Infinite-dimensional groups and their representations, (Lectures at the European School in Group Theory, SDU-Odense Univ., August 2000), preprint, Technische Universität Darmstadt 2206 (2002), April.

[Ne01] - Highest weight representations and infinite-dimensional Kähler manifolds, Recent advances in Lie theory (Vigo, 2000), 367-392. Res. Exp. Math., 25, Heldermann, Lemgo, 2002.

[Pe88] V. G. Pestov, Fermeture nonstandard des algèbres et groupes de Lie banachiques, C. R. Acad. Sci. Paris Sèr. I Math. 306 (1988), 643-645.

[Pe92] - Nonstandard hulls of Banch-Lie groups and algebras, Nova J. Algebra Geom. 1 (1992), 371-381.

[PS90] A. Pressley, G. Segal, Loop groups, (Reprinted with corrections). The Clarendon Press, Oxford University Press, New York, 1990.

[Up85] H. Upmeier, Symmetric Banach manifolds and Jordan $C^{*}$-algebras, North-Holland Mathematics Studies, 104. Notas de Matemática, 96. North-Holland Publishing Co., Amsterdam, 1985.

Institute of Mathematics "Simion Stoilow" of the Romanian Academy, P.O. Box 1-764, RO-70700 Bucharest, Romania.

E-mail address: Daniel.Beltita@imar.ro 